\title{
Hydrogel-Based Controlled Delivery Systems for Articular Cartilage Repair
}

\author{
Ana Rey-Rico, ${ }^{1}$ Henning Madry, ${ }^{1,2}$ and Magali Cucchiarini ${ }^{1}$ \\ ${ }^{1}$ Center of Experimental Orthopaedics, Saarland University Medical Center, Homburg, 66421 Saar, Germany \\ ${ }^{2}$ Department of Orthopaedic Surgery, Saarland University Medical Center, Homburg, 66421 Saar, Germany \\ Correspondence should be addressed to Ana Rey-Rico; ana.rey.rico@gmail.com
}

Received 13 April 2016; Accepted 12 July 2016

Academic Editor: GeunHyung Kim

Copyright (c) 2016 Ana Rey-Rico et al. This is an open access article distributed under the Creative Commons Attribution License, which permits unrestricted use, distribution, and reproduction in any medium, provided the original work is properly cited.

\begin{abstract}
Delivery of bioactive factors is a very valuable strategy for articular cartilage repair. Nevertheless, the direct supply of such biomolecules is limited by several factors including rapid degradation, the need for supraphysiological doses, the occurrence of immune and inflammatory responses, and the possibility of dissemination to nontarget sites that may impair their therapeutic action and raise undesired effects. The use of controlled delivery systems has the potential of overcoming these hurdles by promoting the temporal and spatial presentation of such factors in a defined target. Hydrogels are promising materials to develop delivery systems for cartilage repair as they can be easily loaded with bioactive molecules controlling their release only where required. This review exposes the most recent technologies on the design of hydrogels as controlled delivery platforms of bioactive molecules for cartilage repair.
\end{abstract}

\section{Introduction}

Articular cartilage is an avascular tissue that lines the weightbearing surface of joints, formed of organized populations of chondrocytes surrounded by their extracellular matrix (ECM) (proteoglycans, type II collagen) and regulated by a metabolic balance that involves diverse signaling molecules, growth factors, and cytokines [1]. Due to the lack of access to blood supply, the cartilage has a limited ability to selfheal and full repair of cartilage defects is thus a major clinical challenge that may progress to osteoarthritis [24], a critical disorder affecting a large number of patients. While various options are available to repair injured cartilage (marrow-stimulating techniques, transplantation of tissue or cells as autologous chondrocytes or mesenchymal stem cells (MSCs), and replacement surgery) [5-8], still none of them are capable of reproducing the natural functions of the native, hyaline cartilage, rather leading to the formation of a poorly mechanically functional fibrocartilaginous surface (type I collagen). In this sense, large efforts are ongoing to improve these procedures and considerable progress has been made in the last few years by identifying novel methods and factors that may stimulate the reparative activities in sites of cartilage injury. Nevertheless, regenerating the desirable phenotypic response from host and/or codelivered progenitor cells remains a major issue in orthopaedics.

Tissue engineering and regenerative medicine approaches based on the design of biomaterials scaffolds reflecting the properties of the native cartilage may provide potent alternatives to restore a healthy and fully functional articular cartilage. Thus far, advances in biomedical devices for controlled drug delivery platforms support a new generation of products to treat such disorders where a temporal control over the pharmacokinetic profiles is required [9]. Hence, the use of biomaterials as controlled delivery systems has shown to be a powerful strategy to improve the temporal and spatial presentation of therapeutic agents in a defined target protecting the cargo from physiological degradation $[10,11]$. Most advanced tissue engineering approaches for cartilage repair involve a combination of scaffolds with optimal properties, cells relevant to the tissue (chondrocytes, MSCs), and biological cues (growth factors/cytokines or therapeutic gene transfer vectors). Hydrogel-based biomaterials are promising compounds for cartilage repair not only because 
of their high water content like in the native cartilage but also because they can be typically fabricated under mild conditions, enabling the encapsulation of labile biomolecules such as growth factors [12].

The goal of the present work is to provide an overview of the most recent advances in the preparation of polymeric hydrogel networks as controlled delivery systems of the most relevant bioactive molecules involved in articular cartilage repair.

\section{Hydrogels in Cartilage Tissue Engineering}

2.1. Features of Hydrogels. Hydrogels are polymeric networks consisting of cross-linked hydrophilic polymers able to swell and retain a significant fraction of water within their structure but will not dissolve in water [13]. The ability of hydrogels to absorb water arises from hydrophilic functional groups attached to the polymeric backbone while their resistance to dissolution is a result of the cross-links between network chains. In general, hydrogels are characterized by good biocompatibility, high permeability for oxygen and nutrients, production under mild conditions, and ease of cell encapsulation resulting in a homogeneous distribution [12]. Hydrogels are particularly attractive biomaterials for cartilage repair as they reflect the diverse properties of native cartilage and can be provided in a minimally invasive way to fill defects of any size [14].

Hydrogels can be classified into physical and chemical systems based on their cross-linking mechanism [15, 16]. While physical cross-links involve the entanglement of the chains by hydrogen bonding, hydrophobic interaction, and crystallite formation, chemical (or covalent) cross-links are permanent junctions formed by covalent bonds [16]. Regarding their nature, hydrogels can be categorized as natural, synthetic, or natural/synthetic hybrid biomaterials as a function of the origin from the polymers used for their fabrication [15]. Natural hydrogels have high biocompatibility and inherent biodegradability and are commonly used in cartilage tissue engineering due to intrinsic prochondrogenic properties and involvement in native cellular processes $[16$, 17]. The most widely exploited natural polymers for cartilage tissue engineering include alginate [18-22], hyaluronic acid (HA) [23, 24], and fibrin [25-30]. Synthetic hydrogels have more reproducible physical and chemical properties than natural polymers but they lack such cell bioactivity properties [16]. Synthetic hydrogels can be designed to be "smart" or stimuli-sensitive polymers, having the ability to swell or deswell in response to small changes in the environment such as temperature, $\mathrm{pH}$, or ionic strength [31, 32]. Some of the most extensively used synthetic polymers for cartilage repair include polyester copolymers from poly(lactic acid) as poly(lactide-co-glycolide) (PLGA) [33, 34], selfassembling peptides [35-37], nonbiodegradable polymers as polyethylene glycol (PEG) [38-42], thermoreversible polymers such as poly(N-isopropylacrylamide) (pNIPAAm) [43], and polyethylene oxide (PEO) and polypropylene oxide(PPO-) based copolymers (poloxamers or Pluronic ${ }^{\circledR}$ and poloxamines or Tetronic $\left.{ }^{\circledR}\right)[22,44]$.
2.2. Hydrogels as Controlled Delivery Systems for Cartilage Repair. The use of hydrogels as controlled delivery systems of bioactive molecules in strategies of cartilage repair aims at reproducing the complex microenvironment that naturally occurs in articular cartilage in an artificial setting [45]. Control over delivery of the therapeutic factors can be achieved by tuning physical properties from the hydrogels such as pore size and degradation kinetics [16]. Appropriate pore size and density are key parameters to modulate the release of the bioactive molecules and to ensure the accommodation of viable cells for cartilage tissue engineering approaches, involving the immobilization of cells within the scaffold. Biodegradability of the hydrogels is also another essential feature as it should be orchestrated by the new tissue formation rate.

Controlled delivery systems have been conceived to modify certain parameters of the biomolecule to be delivered including its release profile, ability to cross biological barriers, biodistribution, clearance, and stability (metabolism) [46]. Hence, the therapeutic efficiency of the bioactive molecule will strongly depend on the success rate of the active substances to reach the target site. While the easiest way to achieve cartilage repair is probably based on the direct intra-articular injection of a therapeutic composition, the frequency and (supraphysiological) levels of the therapeutic dose required, plus the possible diffusion of the treatment to nontarget sites and potential neutralization by host inflammatory or immune responses, often hinder the overall efficacy of the approach $[11,47]$.

Another key parameter when designing a hydrogelbased controlled delivery system for cartilage repair is the mechanism of loading of the bioactive substance into the hydrogel network, having a strong influence on its release profile and subsequently on its therapeutic action. Incorporation of bioactive substances within hydrogel networks can be performed by physical encapsulation, physical or chemical immobilization of the biomolecules to the polymeric network, and electrostatic interaction [45]. Physical encapsulation is a mild approach to load a molecule into the polymeric network preventing its denaturalization [48]. By this method, the release profile of the bioactive factor is mainly controlled by diffusion of the molecule through the pores of the hydrogel network but often results in a short-term release. Chemical or physical immobilization of biomolecules in a hydrogel network involves a release profile controlled by polymer degradation, linker, or by dissociation from the gel matrix. Even though a long-term release profile of the therapeutic molecule is usually achieved with this method, the harsh conditions involved here to immobilize the molecule within the hydrogel network can produce its denaturalization. Thus far, strong chemical bonds can lead to an incomplete release of the molecule from the hydrogel $[45,49]$.

An overview of the most relevant strategies used for the fabrication of hydrogels for controlled release of bioactive substances for cartilage repair is presented in the following section (Figure 1). 


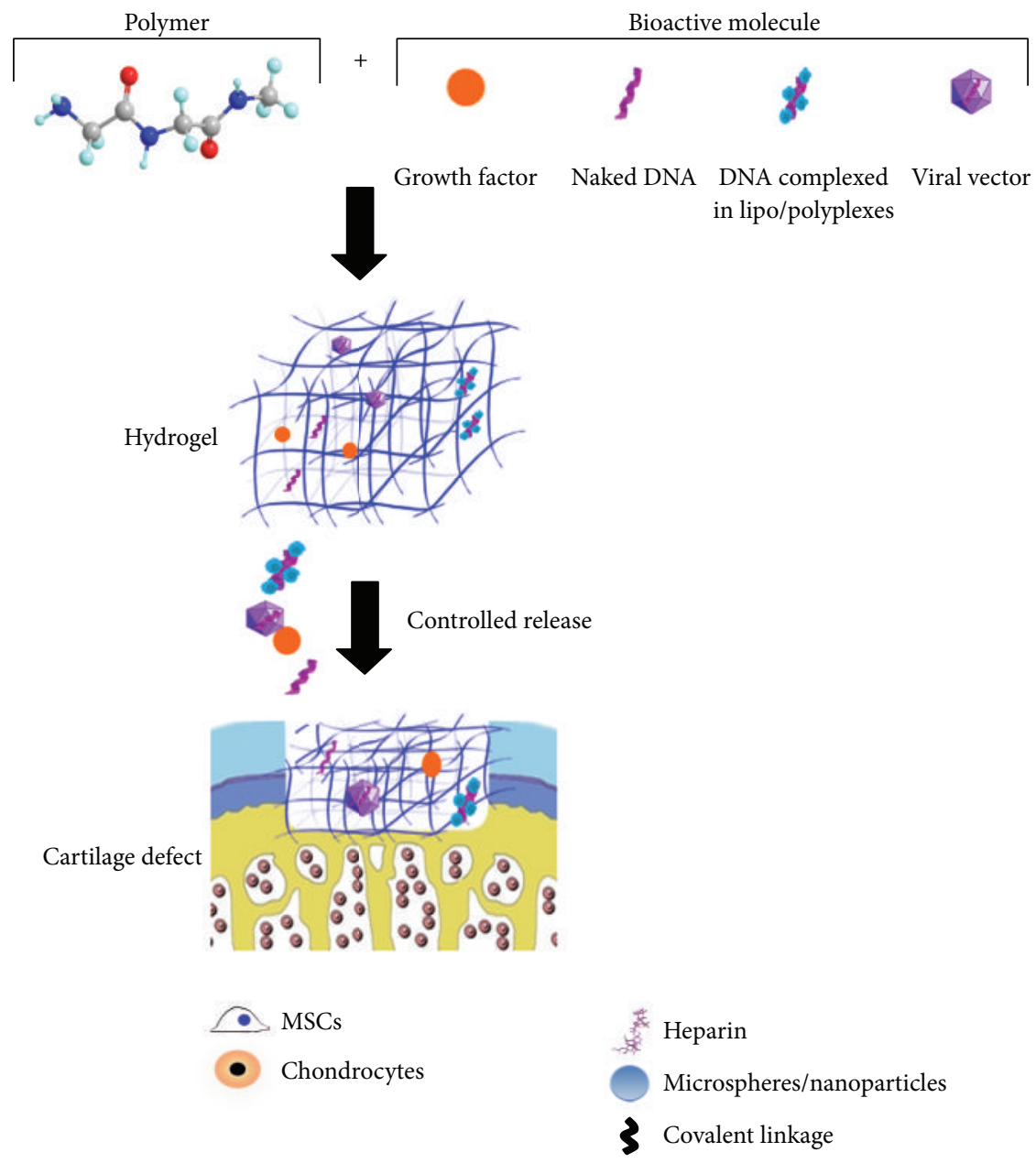

FIGURE 1: Overview of the main strategies used to design hydrogel-based delivery systems for cartilage repair. Chondroreparative factors (growth factors, nonviral gene transfer vectors including naked DNA or DNA complexed in lipo/polyplexes, and viral gene transfer vectors) may be encapsulated or immobilized into hydrogel networks by exploiting different properties from the biomolecule itself, as the affinity for heparin from growth factors. Delivery systems can be endowed with relevant populations for cartilage repair such as MSCs and chondrocytes or directly implanted as cell-free constructs into the cartilage defects providing a sustained release profile of the therapeutic factor.

\section{Controlled Delivery of Bioactive Factors from Hydrogels for Cartilage Repair}

Hydrogel-based delivery systems have been used for the controlled release of bioactive factors, having a pivotal role in cartilage regeneration processes such as growth factors [12] and gene transfer vectors [47].

3.1. Growth Factors. Growth factors are polypeptides involved in the cellular communication system, capable of transmitting signals that modulate cellular activity by either stimulating or inhibiting cellular proliferation, migration, differentiation, and/or gene expression [50, 51]. Growth factors have a pleiotropic nature; that is, the same growth factor may act on different cell types inducing similar or different effects. Growth factors are usually produced by the cells as inactive or partially active precursors. They can be activated upon proteolytic cleavage or by binding to the ECM $[50,51]$. Hence, growth factors must be delivered into the target place to be active as their rapid inactivation in physiological conditions (half-life in range of minutes) compromises their capacity to reach the cellular ECM. As a matter of fact, single doses of growth factors are often ineffective and large supraphysiological doses with the subsequent risks of severe adverse effects are required to initiate the healing of cartilage defects $[12,52,53]$. Thus, the use of hydrogels that provide a temporal or spatiotemporal control of the growth factor may be a valuable strategy to circumvent such limitations. Among the most widely used growth factors involved in cartilage repair are members of the transforming growth factor $(\mathrm{TGF}-\beta)$ superfamily [12] or bone morphogenetic proteins (BMP) [54], basic fibroblast growth factor (FGF-2) [55], and insulin-like growth factor I (IGF-I) [56].

Controlled delivery of growth factors from hydrogels may occur over an extended time, reducing the need for additional applications of the protein. Moreover, strictly localized release of growth factors may confine their activity to a distinct location in the proximity of the defect site 
reducing potential side effects [57]. Delivery of growth factors from hydrogels can be modulated by tuning different properties from the biomaterial as cross-linking density changes the free space for diffusion of the bioactive molecule [58]. Other strategies include the modification of the interaction of the growth factor with the hydrogel network as charge interactions [58, 59]. Controlled release of growth factors for cartilage repair focuses on the targeting of relevant cell populations involved in cartilage regenerative processes, specifically chondrocytes and MSCs. A compilation of the main biomaterials used to produce hydrogels in cartilage repair as well of the strategies involved to incorporate the growth factors and their release profile from the polymeric network is given in Table 1 .

Natural polymers such as alginate $[18,19]$, fibrin [2528, 30], HA [23, 24], and chitosan [60] have been widely used to produce hydrogel-based delivery systems of growth factors due to the optimal properties from these biomaterials mimicking the natural ECM from cartilage. Encapsulation into fibrin hydrogels is a valuable strategy to develop controlled delivery systems for cartilage repair as diffusion from the biomolecules can be modulated by modifying some parameters from the hydrogel network as fibrinogen component or thrombin concentration [26] and has been used for controlled release of IGF-I [25, 27] and TGF- $\beta 1$ [26] both in vitro to target MSCs [26] or in an osteochondral defects model in vivo $[25,27]$, showing effectiveness in promoting cartilage regenerative processes. An advantageous strategy for controlling the delivery of growth factors involves the heparinization of hydrogel to immobilize the growth factor by binding to their heparin-binding domains that enable linkage with their receptors [61]. This strategy has been used for immobilization of TGF- $\beta 1$ [28] or BMP-2 [23] in fibrin [28] and HA [23] hydrogels.

Synthetic polymers such as PEG [33, 38-40, 42], selfassembling peptides [35, 36], pNIPAAm [43], and poly(vinyl alcohol) (PVA) [34] have been used to prepare hydrogels as delivery systems of growth factors in cartilage tissue engineering approaches. PEG is the most investigated polymer for hydrogel production due to its good solubility in water and in organic solvents and lack of toxicity [33, 38-40, 42]. Incorporation of growth factor loaded in microspheres into interconnected PEG-based hydrogels is an attractive strategy to simultaneously achieve a sustained release of the protein and an adequate microenvironment for chondrogenesis [62]. Park et al. [39] embedded bovine chondrocytes into oligo(poly(ethylene glycol) fumarate) (OPF) composite hydrogels coencapsulating gelatin microparticles loaded with TGF- $\beta 1$. Controlled release of TGF- $\beta 1$ from the constructs increased cellularity with maintenance of the cell phenotype [39].

Most advanced strategies focused on the dual release of growth factors to achieve a synergistic effect on the enhancement of chondrogenic differentiation and on the maintenance of their phenotype. Holland et al. [40] investigated the local delivery of TGF- $\beta 1$ and IGF-I incorporated into biocompatible hydrogels based on OPF with gelatin microparticles. When delivered to osteochondral defects in rabbits, the best histological result was observed after
3 months in vivo with IGF-I-treated defects, while these benefits were not maintained when codelivered with TGF$\beta 1$ or when TGF- $\beta 1$ was delivered alone, suggesting that in this in vivo model IGF-I was superior to TGF- $\beta 1$ [40]. In the most recent work, the same authors found that while delivery of BMP-2 enhanced subchondral bone formation, dual delivery of IGF-I and BMP-2 in separate layers did not improve cartilage repair but they may synergistically enhance the degree of subchondral bone formation [42].

3.2. Nonviral Gene Delivery of Factors. Gene transfer via nonviral vectors (transfection) is based on the incorporation of DNA, either naked or complexed with cationic polymers or with cationic lipids (in polyplexes and lipoplexes), into the target population. Though considered as a safe method as it avoids the risk of acquiring replication competence and of insertional mutagenesis without inducing immune responses in the host, its use is limited by the low transfection efficiencies (40-50\% maximum) and short-term transgene expression levels achieved [63-65]. While the use of hydrogels for controlled delivery of growth factors has been broadly exploited in the context of cartilage repair, their application as controlled delivery systems of gene transfer vectors has been mainly focused on the improvement of the efficiency of transfection and the durability of the expression of transgene in tissue engineering approaches in general, with a very limited number of studies reporting their use on cartilage repair [41]. An overview of the main materials used for the fabrication of hydrogels for controlled delivery of nonviral vectors is summarized in Table 2.

Local gene delivery via hydrogel scaffolds has been studied through the encapsulation of naked DNA during hydrogel formation $[66,67]$ using synthetic polymers such as PLGA [66] or pNIPAAm [67]. Although naked DNA achieved gene expression and guided repair in vivo [76], its low gene transfer efficiency and rapid diffusion of the DNA from the hydrogel network urged searching for alternative gene delivery systems like those based on the controlled release of DNA complexed in lipoplexes or polyplexes [74]. Gene delivery of polyplexes $[41,70-75]$ or lipoplexes $[68,69]$ has been studied by using different hydrogel systems including fibrin [69, 72], HA [70, 72-75], and PEG [41, 68, 70, 71] to target different cell populations including MSCs $[71,74,75]$ in a variety of tissue engineering approaches (Table 2).

An important limitation of the incorporation of nonviral gene transfer vectors into hydrogels is the failure of loading high DNA concentrations because of their tendency to aggregate. Incorporation of nonviral vectors into hydrogel networks often results in their aggregation as a result of the soft, loose, and charged structures of polyplexes and lipoplexes [70,73]. To solve such issues, a caged nanoparticle encapsulation technology has been designed [70, 73, 74]. Incorporation of lyophilized powder of polyplexes suspended in a medium containing sucrose and agarose into HA hydrogels significantly prevented their aggregation compared with direct encapsulation of DNA/polyethylene imine (PEI) control polyplexes $[70,73,74]$. 


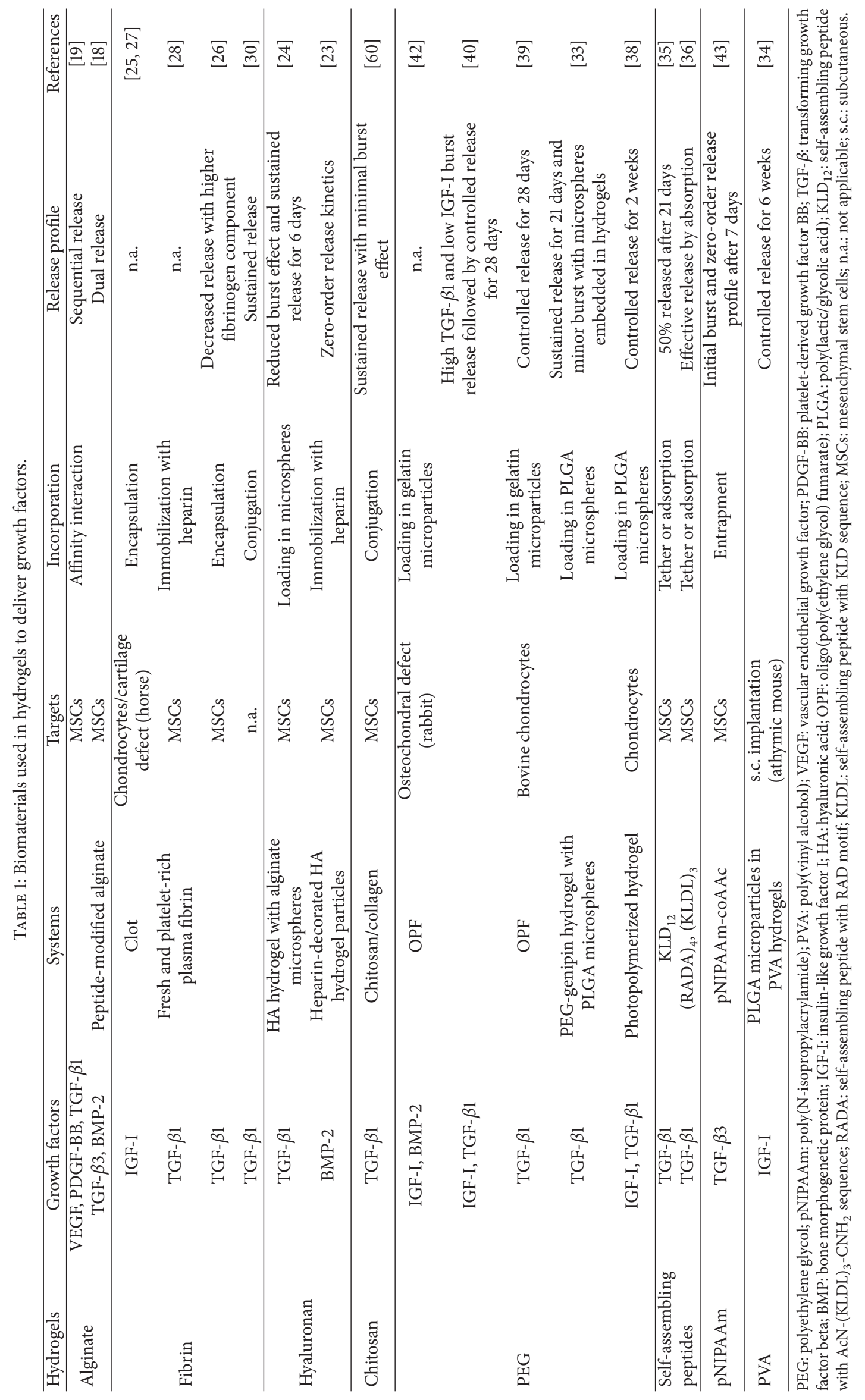




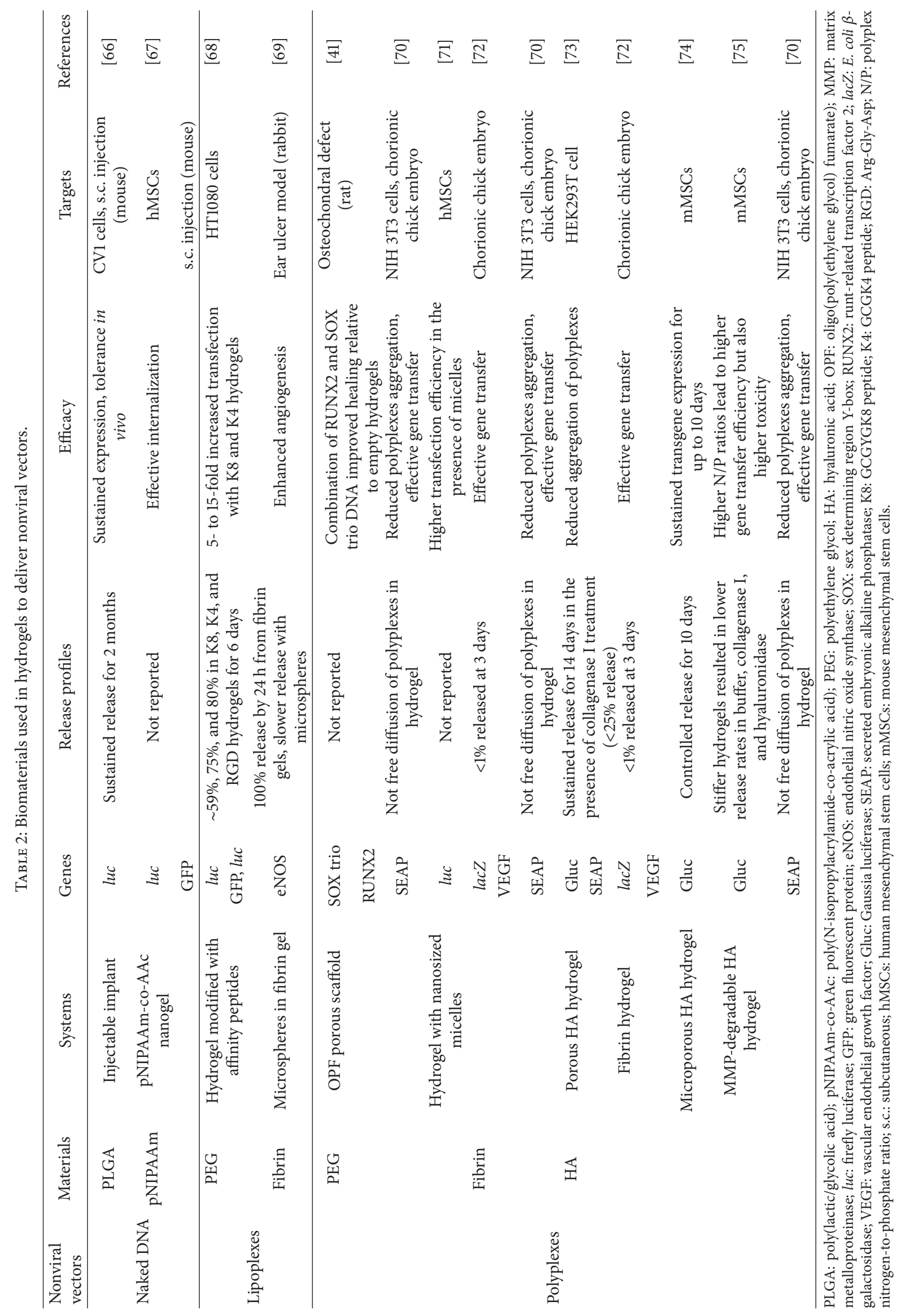


The use of hydrogels as controlled gene delivery systems for cartilage repair is a very valuable but still developing strategy. Osteochondral and chondral units are especially promising tissues for polymeric gene delivery approaches because of the limited blood flow to the region which could cause problems in DNA polymer complex delivery and the potential for the delivered genes to induce differentiation of infiltrated MSCs [41]. Needham et al. [41] recently described an innovative approach by delivering DNA polyplexes from OPF hydrogel scaffolds for osteochondral injury repair. An OPF layered scaffold mimicking the native osteochondral tissue organization was simultaneously loaded with DNA polyplexes encoding the runt-related transcription factor 2 (RUNX2) or sex determining region Y-box 5, 6, and 9 (SOX trio) to generate bone and cartilage tissues, respectively, in a rat osteochondral defect model. At 6 weeks after implantation, combination of RUNX2 and SOX trio DNA led to a significantly improved healing ability compared with empty hydrogels or each factor alone [41].

3.3. Viral Gene Delivery of Factors. Gene transfer using viral vectors (transduction) is based on the natural cellular entry pathways of viruses from which they are derived, resulting in higher gene transfer efficiencies compared with nonviral vectors $(80-90 \%)[47,65,77,78]$. The most common viruses manipulated for gene transfer purposes include adenoviruses [79-81], herpes simplex virus (HSV) [82], retro- and lentiviruses [83-85], and adeno-associated virus (AAV) [8689]. Although gene transfer via viral vectors is highly efficient, the existence of patient-associated factors and physiological barriers (existence of neutralizing antibodies against the viral capsid and inhibition of transduction in the presence of specific anticoagulants) may interfere with the effective delivery, processing, and expression of transgene inside the target cells $[47,90]$. Also, intra-articular injection of viral vectors can result in the rapid dispersion of the particles from the joint space and diffusion to nontarget sites, leading to reduced gene transfer efficiencies in cells recruited in the lesions $[29,91]$.

Controlled release of viral vectors from hydrogels allowing for a release pattern via a diffusion process may help overcome these issues. A summary of the main biomaterials used to produce hydrogels for controlled release of viral vectors in different tissue engineering approaches is shown in Table 3. Most of the work reporting the use of hydrogels as controlled delivery systems of viral vectors aimed at overcoming the limitations associated with these types of vectors in different tissue engineering approaches, with only a few publications focusing on cartilage repair [22, 29, 37].

Adenoviral, lentiviral, and rAAV vectors have been encapsulated in fibrin hydrogels, taking advantage of the low immunogenicity and biodegradability of this compound and showing sustained release profiles of the vectors and expression of the transgenes of interest in different cell targets [29, 93-95]. Most interestingly, Lee et al. [29] reported that release of an rAAV carrying TGF- $\beta$ from diluted fibrin glue hydrogels resulted in enhanced production of TGF- $\beta$ and higher levels of cartilage-specific gene expression in human
MSCs (hMSCs) compared with undiluted hydrogels. This fact was attributed to the more open network structure from diluted fibrin glue hydrogels compared with undiluted ones resulting in the most efficient release of rAAV TGF- $\beta$ vectors [29].

Self-assembling peptides RAD16-I in a pure (RAD) form or combined with HA (RAD-HA) have been also employed to release $\mathrm{rAAV}$ vectors as a means of genetically modifying hMSCs [37]. Such systems were capable of efficiently encapsulating and releasing rAAV in a sustained, controlled manner to effectively transduce the cells (up to 80\%) without deleterious effects on cell viability (up to 100\%) or on their potential for chondrogenic differentiation of the cells over time (up to 21 days) [37].

PEO- and PPO-based "smart" or "intelligent" selfassembling, temperature-sensitive copolymers have been also utilized as efficient rAAV-mediated delivery systems due to their capacity to form polymeric micelles and to undergo solto-gel transition upon heating $[96,97]$. Specifically, encapsulation of rAAV vectors in poloxamer PF68 and poloxamine T908 polymeric micelles allowed for effective, durable, and safe modification of hMSCs via rAAV to levels similar to or even higher than those noted upon direct vector application (up to 95\% of gene transfer efficiency) [44]. Of further note, these copolymers were capable of restoring the transduction of hMSCs with rAAV in conditions of gene transfer inhibition like in the presence of heparin or of a specific antibody directed against the rAAV capsid, enabling effective therapeutic delivery of a chondrogenic sox 9 sequence leading to enhanced chondrocyte differentiation of the cells [44]. Furthermore, various hydrogel composite structures based on alginate (AlgPH155) and poloxamer PF127 were prepared by cross-linking at either high $\left(50^{\circ} \mathrm{C}\right.$; AlgPH155+PF127 $\left.[\mathrm{H}]\right)$ or room temperature (AlgPH155+PF127 [C]) to encapsulate and release rAAV vectors [22]. Strikingly, hydrogels based on AlgPH155 alone had the highest initial burst of rAAV release while those cross-linked as AlgPH155+PF127 [C] had the most sustained release pattern, all leading to high transduction efficiencies in hMSCs $(\sim 80 \%)$ over an extended period of evaluation (up to 21 days) [22].

\section{Conclusions and Outlook}

The use of hydrogels as controlled delivery systems of bioactive molecules is a valuable strategy to achieve appropriate levels of a therapeutic factor into target places, circumventing possible limitations associated with their direct administration (frequency and amounts of required doses, inflammatory and host immune responses, and possible diffusion to nontarget locations). Hydrogel delivery platforms can be modulated by tuning some specific parameters from the hydrogel networks as composition, cross-linking density pore size, and degradation kinetics. Hydrogel networks are promising systems for cartilage tissue engineering approaches as they exhibit many intrinsic features mimicking the ECM from cartilage showing in many studies biocompatibility with key cell populations involved in cartilage regenerative processes as chondrocytes and MSCs. 


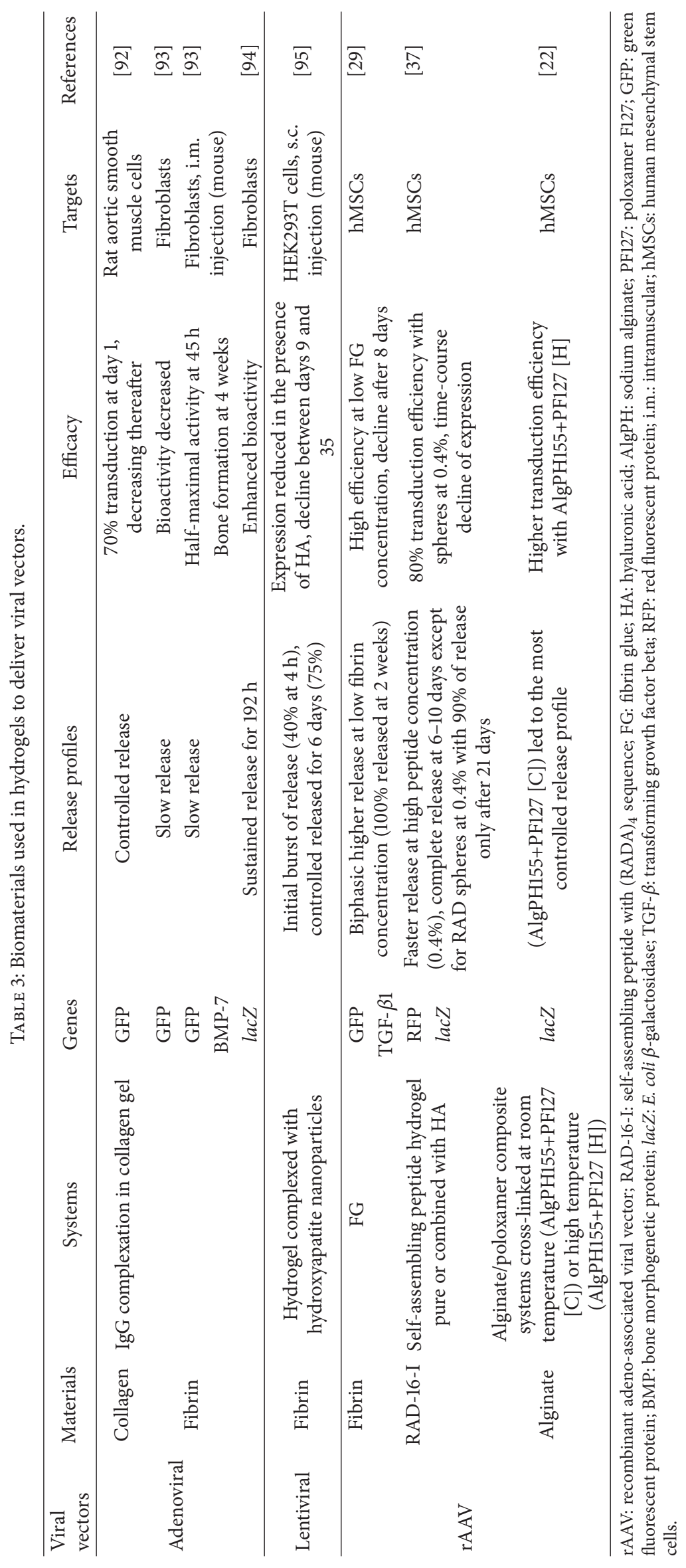


Although promising technological advances have been reported to produce hydrogels as delivery systems of cartilage reparative factors, large efforts are still necessary to obtain adapted hydrogel-based delivery systems that may lead to successful clinical translation in patients integrating conditions of efficacy, durability, and safety. In this sense, many relevant parameters from the hydrogel platforms such as encapsulation efficiency, interaction with and stability of the biomolecule cargo, tensile strength, resistance against dilution, gene transfer efficiency, and biocompatibility need to be accurately optimized. It will be also very important to keep in mind that a successful system in vitro might not generate similar/sufficient or adapted effects in a native environment in vivo. It will thus be fundamental to test the adaptability of the hydrogel systems in sites of tissue damage using clinically relevant, complex orthotopic animal models of cartilage defect as a means of enhancing the natural repair processes. Yet, despite remaining challenges, recent advances in the development of hydrogels as controlled release delivery systems of cartilage reparative factors are promising, new avenues of research that may clearly improve cartilage repair in patients in a close future.

\section{Competing Interests}

The authors declare that they have no competing interests.

\section{Acknowledgments}

This work was supported by a grant from Deutsche Forschungsgemeinschaft (DFG RE 328/2-1 to Ana Rey-Rico, Magali Cucchiarini, and Henning Madry).

\section{References}

[1] M. Cucchiarini and H. Madry, "Genetic modification of mesenchymal stem cells for cartilage repair," Bio-Medical Materials and Engineering, vol. 20, no. 3-4, pp. 135-143, 2010.

[2] M. B. Goldring and S. R. Goldring, "Osteoarthritis," Journal of Cellular Physiology, vol. 213, no. 3, pp. 626-634, 2007.

[3] L. J. Sandell, "Etiology of osteoarthritis: genetics and synovial joint development," Nature Reviews Rheumatology, vol. 8, no. 2, pp. 77-89, 2012.

[4] B. Johnstone, M. Alini, M. Cucchiarini et al., "Tissue engineering for articular cartilage repair-the state of the art," European Cells and Materials, vol. 25, pp. 248-267, 2012.

[5] M. Brittberg, A. Lindahl, A. Nilsson, C. Ohlsson, O. Isaksson, and L. Peterson, "Treatment of deep cartilage defects in the knee with autologous chondrocyte transplantation," The New England Journal of Medicine, vol. 331, no. 14, pp. 889-895, 1994.

[6] U. Horas, D. Pelinkovic, G. Herr, T. Aigner, and R. Schnettler, "Autologous chondrocyte implantation and osteochondral cylinder transplantation in cartilage repair of the knee joint. A prospective, comparative trial," The Journal of Bone \& Joint Surgery-American Volume, vol. 85, no. 2, pp. 185-192, 2003.

[7] G. Bentley, L. C. Biant, R. W. J. Carrington et al., "A prospective, randomised comparison of autologous chondrocyte implantation versus mosaicplasty for osteochondral defects in the knee," Journal of Bone and Joint Surgery Series B, vol. 85, no. 2, pp. 223230, 2003.
[8] G. Knutsen, L. Engebretsen, T. C. Ludvigsen et al., "Autologous chondrocyte implantation compared with microfracture in the knee. A randomized trial," The Journal of Bone and Joint Surgery American, vol. 86, no. 3, pp. 455-464, 2004.

[9] P. Gurman, O. R. Miranda, K. Clayton, Y. Rosen, and N. M. Elman, "Clinical applications of biomedical microdevices for controlled drug delivery," Mayo Clinic Proceedings, vol. 90, no. 1, pp. 93-108, 2015.

[10] V. Pillay, A. Seedat, Y. E. Choonara, L. C. du Toit, P. Kumar, and V. M. K. Ndesendo, "A review of polymeric refabrication techniques to modify polymer properties for biomedical and drug delivery applications," AAPS PharmSciTech, vol. 14, no. 2, pp. 692-711, 2013.

[11] J. Lam, S. Lu, F. K. Kasper, and A. G. Mikos, "Strategies for controlled delivery of biologics for cartilage repair," Advanced Drug Delivery Reviews, vol. 84, pp. 123-134, 2015.

[12] H. Madry, A. Rey-Rico, J. K. Venkatesan, B. Johnstone, and M. Cucchiarini, "Transforming growth factor beta-releasing scaffolds for cartilage tissue engineering," Tissue Engineering B: Reviews, vol. 20, no. 2, pp. 106-125, 2014.

[13] E. M. Ahmed, "Hydrogel: preparation, characterization, and applications: a review," Journal of Advanced Research, vol. 6, no. 2, pp. 105-121, 2015.

[14] C. Chung and J. A. Burdick, "Engineering cartilage tissue," Advanced Drug Delivery Reviews, vol. 60, no. 2, pp. 243-262, 2008.

[15] B. V. Slaughter, S. S. Khurshid, O. Z. Fisher, A. Khademhosseini, and N. A. Peppas, "Hydrogels in regenerative medicine," Advanced Materials, vol. 21, no. 32-33, pp. 3307-3329, 2009.

[16] J. Zhu and R. E. Marchant, "Design properties of hydrogel tissue-engineering scaffolds," Expert Review of Medical Devices, vol. 8, no. 5, pp. 607-626, 2011.

[17] I. L. Kim, R. L. Mauck, and J. A. Burdick, "Hydrogel design for cartilage tissue engineering: a case study with hyaluronic acid," Biomaterials, vol. 32, no. 34, pp. 8771-8782, 2011.

[18] C. A. Simmons, E. Alsberg, S. Hsiong, W. J. Kim, and D. J. Mooney, "Dual growth factor delivery and controlled scaffold degradation enhance in vivo bone formation by transplanted bone marrow stromal cells," Bone, vol. 35, no. 2, pp. 562-569, 2004.

[19] I. Freeman and S. Cohen, "The influence of the sequential delivery of angiogenic factors from affinity-binding alginate scaffolds on vascularization," Biomaterials, vol. 30, no. 11, pp. 2122-2131, 2009.

[20] T. Re'em, Y. Kaminer-Israeli, E. Ruvinov, and S. Cohen, "Chondrogenesis of hMSC in affinity-bound TGF- $\beta$ scaffolds," Biomaterials, vol. 33, no. 3, pp. 751-761, 2012.

[21] J. A. Simson, I. A. Strehin, Q. Lu, M. O. Uy, and J. H. Elisseeff, "An adhesive bone marrow scaffold and bone morphogenetic2 protein carrier for cartilage tissue engineering," Biomacromolecules, vol. 14, no. 3, pp. 637-643, 2013.

[22] P. Díaz-Rodríguez, A. Rey-Rico, H. Madry, M. Landin, and M. Cucchiarini, "Effective genetic modification and differentiation of hMSCs upon controlled release of rAAV vectors using alginate/poloxamer composite systems," International Journal of Pharmaceutics, vol. 496, no. 2, pp. 614-626, 2015.

[23] X. Xu, A. K. Jha, R. L. Duncan, and X. Jia, "Heparin-decorated, hyaluronic acid-based hydrogel particles for the controlled release of bone morphogenetic protein 2," Acta Biomaterialia, vol. 7, no. 8, pp. 3050-3059, 2011. 
[24] L. Bian, D. Y. Zhai, E. Tous, R. Rai, R. L. Mauck, and J. A. Burdick, "Enhanced MSC chondrogenesis following delivery of TGF- $\beta 3$ from alginate microspheres within hyaluronic acid hydrogels in vitro and in vivo," Biomaterials, vol. 32 , no. 27 , pp. 6425-6434, 2011.

[25] A. J. Nixon, L. A. Fortier, J. Williams, and H. Mohammed, "Enhanced repair of extensive articular defects by insulinlike growth factor-I-laden fibrin composites," Journal of Orthopaedic Research, vol. 17, no. 4, pp. 475-487, 1999.

[26] I. Catelas, J. F. Dwyer, and S. Helgerson, "Controlled release of bioactive transforming growth factor beta-1 from fibrin gels in vitro," Tissue Engineering Part C: Methods, vol. 14, no. 2, pp. 119$128,2008$.

[27] L. A. Fortier, H. O. Mohammed, G. Lust, and A. J. Nixon, "Insulin-like growth factor-I enhances cell-based repair of articular cartilage," Journal of Bone and Joint Surgery B, vol. 84, no. 2, pp. 276-288, 2002.

[28] T. A. E. Ahmed, A. Giulivi, M. Griffith, and M. Hincke, "Fibrin glues in combination with mesenchymal stem cells to develop a tissue-engineered cartilage substitute," Tissue EngineeringPart A, vol. 17, no. 3-4, pp. 323-335, 2011.

[29] H. H. Lee, A. M. Haleem, V. Yao, J. Li, X. Xiao, and C. R. Chu, "Release of bioactive adeno-associated virus from fibrin scaffolds: effects of fibrin glue concentrations," Tissue Engineering Part A, vol. 17, no. 15-16, pp. 1969-1978, 2011.

[30] M.-S. Liang and S. T. Andreadis, "Engineering fibrin-binding TGF- $\beta 1$ for sustained signaling and contractile function of MSC based vascular constructs," Biomaterials, vol. 32, no. 33, pp. 8684-8693, 2011.

[31] B. Jeong and A. Gutowska, "Lessons from nature: stimuliresponsive polymers and their biomedical applications," Trends in Biotechnology, vol. 20, no. 7, pp. 305-311, 2002.

[32] J. M. Knipe and N. A. Peppas, "Multi-responsive hydrogels for drug delivery and tissue engineering applications," Regenerative Biomaterials, vol. 1, no. 1, pp. 57-65, 2014.

[33] A. J. DeFail, C. R. Chu, N. Izzo, and K. G. Marra, "Controlled release of bioactive TGF- $\beta 1$ from microspheres embedded within biodegradable hydrogels," Biomaterials, vol. 27, no. 8, pp. 1579-1585, 2006.

[34] K. L. Spiller, Y. Liu, J. L. Holloway et al., "A novel method for the direct fabrication of growth factor-loaded microspheres within porous nondegradable hydrogels: controlled release for cartilage tissue engineering," Journal of Controlled Release, vol. 157, no. 1, pp. 39-45, 2012.

[35] P. W. Kopesky, E. J. Vanderploeg, J. D. Kisiday, D. D. Frisbie, J. D. Sandy, and A. J. Grodzinsky, "Controlled delivery of transforming growth factor $\beta 1$ by self-assembling peptide hydrogels induces chondrogenesis of bone marrow stromal cells and modulates smad2/3 signaling," Tissue Engineering_Part A, vol. 17, no. 1-2, pp. 83-92, 2011.

[36] R. E. Miller, P. W. Kopesky, and A. J. Grodzinsky, "Growth factor delivery through self-assembling peptide scaffolds," Clinical Orthopaedics and Related Research, vol. 469, no. 10, pp. 27162724, 2011.

[37] A. Rey-Rico, J. K. Venkatesan, J. Frisch et al., "Effective and durable genetic modification of human mesenchymal stem cells via controlled release of rAAV vectors from self-assembling peptide hydrogels with a maintained differentiation potency," Acta Biomaterialia, vol. 18, pp. 118-127, 2015.
[38] J. Elisseeff, W. McIntosh, K. Fu, T. Blunk, and R. Langer, "Controlled-release of IGF-I and TGF- $\beta 1$ in a photopolymerizing hydrogel for cartilage tissue engineering," Journal of Orthopaedic Research, vol. 19, no. 6, pp. 1098-1104, 2001.

[39] H. Park, J. S. Temenoff, T. A. Holland, Y. Tabata, and A. G. Mikos, "Delivery of TGF- $\beta 1$ and chondrocytes via injectable, biodegradable hydrogels for cartilage tissue engineering applications," Biomaterials, vol. 26, no. 34, pp. 7095-7103, 2005.

[40] T. A. Holland, E. W. H. Bodde, V. M. J. I. Cuijpers et al., "Degradable hydrogel scaffolds for in vivo delivery of single and dual growth factors in cartilage repair," Osteoarthritis and Cartilage, vol. 15, no. 2, pp. 187-197, 2007.

[41] C. J. Needham, S. R. Shah, R. L. Dahlin et al., "Osteochondral tissue regeneration through polymeric delivery of DNA encoding for the SOX trio and RUNX2," Acta Biomaterialia, vol. 10, no. 10, pp. 4103-4112, 2014.

[42] S. Lu, J. Lam, J. E. Trachtenberg et al., "Dual growth factor delivery from bilayered, biodegradable hydrogel composites for spatially-guided osteochondral tissue repair," Biomaterials, vol. 35, no. 31, pp. 8829-8839, 2014.

[43] J. S. Park, D. G. Woo, H. N. Yang et al., "Chondrogenesis of human mesenchymal stem cells encapsulated in a hydrogel construct: neocartilage formation in animal models as both mice and rabbits," Journal of Biomedical Materials Research Part A, vol. 92, no. 3, pp. 988-996, 2010.

[44] A. Rey-Rico, J. K. Venkatesan, J. Frisch et al., "PEO-PPO-PEO micelles as effective rAAV-mediated gene delivery systems to target human mesenchymal stem cells without altering their differentiation potency," Acta Biomaterialia, vol. 27, pp. 42-52, 2015.

[45] R. Censi, A. Dubbini, and P. Matricardi, "Bioactive hydrogel scaffolds-advances in cartilage regeneration through controlled drug delivery," Current Pharmaceutical Design, vol. 21, no. 12, pp. 1545-1555, 2015.

[46] G. Vilar, J. Tulla-Puche, and F. Albericio, "Polymers and drug delivery systems," Current Drug Delivery, vol. 9, no. 4, pp. 367394, 2012.

[47] A. Rey-Rico and M. Cucchiarini, "Controlled release strategies for rAAV-mediated gene delivery," Acta Biomaterialia, vol. 29, pp. 1-10, 2016.

[48] S. K. Seidlits, R. M. Gower, J. A. Shepard, and L. D. Shea, "Hydrogels for lentiviral gene delivery," Expert Opinion on Drug Delivery, vol. 10, no. 4, pp. 499-509, 2013.

[49] J. Sohier, L. Moroni, C. van Blitterswijk, K. de Groot, and J. M. Bezemer, "Critical factors in the design of growth factor releasing scaffolds for cartilage tissue engineering," Expert Opinion on Drug Delivery, vol. 5, no. 5, pp. 543-566, 2008.

[50] M. E. Nimni, "Polypeptide growth factors: targeted delivery systems," Biomaterials, vol. 18, no. 18, pp. 1201-1225, 1997.

[51] K. Lee, E. A. Silva, and D. J. Mooney, "Growth factor deliverybased tissue engineering: general approaches and a review of recent developments," Journal of the Royal Society Interface, vol. 8, no. 55, pp. 153-170, 2011.

[52] L. A. Fortier, J. U. Barker, E. J. Strauss, T. M. McCarrel, and B. J. Cole, "The role of growth factors in cartilage repair," Clinical Orthopaedics and Related Research, vol. 469, no. 10, pp. 27062715, 2011.

[53] E. J. Carragee, E. L. Hurwitz, and B. K. Weiner, "A critical review of recombinant human bone morphogenetic protein-2 trials in spinal surgery: emerging safety concerns and lessons learned," Spine Journal, vol. 11, no. 6, pp. 471-491, 2011. 
[54] A. O. Oshin and M. C. Stewart, "The role of bone morphogenetic proteins in articular cartilage development, homeostasis and repair," Veterinary and Comparative Orthopaedics and Traumatology, vol. 20, no. 3, pp. 151-158, 2007.

[55] M. B. Ellman, D. Yan, K. Ahmadinia, D. Chen, H. S. An, and H. J. Im, "Fibroblast growth factor control of cartilage homeostasis," Journal of Cellular Biochemistry, vol. 114, no. 4, pp. 735-742, 2013.

[56] C. Schmid, "Insulin-like growth factors," Cell Biology International, vol. 19, no. 5, pp. 445-457, 1995.

[57] J. K. Tessmar and A. M. Göpferich, "Matrices and scaffolds for protein delivery in tissue engineering," Advanced Drug Delivery Reviews, vol. 59, no. 4-5, pp. 274-291, 2007.

[58] J. L. Drury and D. J. Mooney, "Hydrogels for tissue engineering: scaffold design variables and applications," Biomaterials, vol. 24, no. 24, pp. 4337-4351, 2003.

[59] K. L. Spiller, S. A. Maher, and A. M. Lowman, "Hydrogels for the repair of articular cartilage defects," Tissue Engineering Part B: Reviews, vol. 17, no. 4, pp. 281-299, 2011.

[60] J. Kim, B. Lin, S. Kim, B. Choi, D. Evseenko, and M. Lee, "TGF$\beta 1$ conjugated chitosan collagen hydrogels induce chondrogenic differentiation of human synovium-derived stem cells," Journal of Biological Engineering, vol. 9, article 1, 2015.

[61] S. Seo and K. Na, "Mesenchymal stem cell-based tissue engineering for chondrogenesis," Journal of Biomedicine and Biotechnology, vol. 2011, Article ID 806891, 8 pages, 2011.

[62] Y. Tabata, "The importance of drug delivery systems in tissue engineering," Pharmaceutical Science and Technology Today, vol. 3, no. 3, pp. 80-89, 2000.

[63] R. S. Goomer, T. M. Maris, R. Gelberman, M. Boyer, M. Silva, and D. Amiel, "Nonviral in vivo gene therapy for tissue engineering of articular cartilage and tendon repair," Clinical Orthopaedics and Related Research, vol. 379, supplement, pp. S189-S200, 2000.

[64] H. Madry, G. Kaul, M. Cucchiarini et al., "Enhanced repair of articular cartilage defects in vivo by transplanted chondrocytes overexpressing insulin-like growth factor I (IGF-I)," Gene Therapy, vol. 12, no. 15, pp. 1171-1179, 2005.

[65] A. Saraf and A. G. Mikos, "Gene delivery strategies for cartilage tissue engineering," Advanced Drug Delivery Reviews, vol. 58, no. 4, pp. 592-603, 2006.

[66] R. E. Eliaz and F. C. Szoka Jr., "Robust and prolonged gene expression from injectable polymeric implants," Gene Therapy, vol. 9, no. 18, pp. 1230-1237, 2002.

[67] J. S. Park, H. N. Yang, D. G. Woo, S. Y. Jeon, and K.-H. Park, "Poly(N-isopropylacrylamide-co-acrylic acid) nanogels for tracing and delivering genes to human mesenchymal stem cells," Biomaterials, vol. 34, no. 34, pp. 8819-8834, 2013.

[68] J. A. Shepard, P. J. Wesson, C. E. Wang et al., "Gene therapy vectors with enhanced transfection based on hydrogels modified with affinity peptides," Biomaterials, vol. 32 , no. 22, pp. 50925099, 2011.

[69] M. M. Kulkarni, U. Greiser, T. O’Brien, and A. Pandit, "A temporal gene delivery system based on fibrin microspheres," Molecular Pharmaceutics, vol. 8, no. 2, pp. 439-446, 2011.

[70] Y. Lei, S. Huang, P. Sharif-Kashani, Y. Chen, P. Kavehpour, and T. Segura, "Incorporation of active DNA/cationic polymer polyplexes into hydrogel scaffolds," Biomaterials, vol. 31, no. 34, pp. 9106-9116, 2010.

[71] Y. Li, C. Yang, M. Khan et al., "Nanostructured PEG-based hydrogels with tunable physical properties for gene delivery to human mesenchymal stem cells," Biomaterials, vol. 33, no. 27, pp. 6533-6541, 2012.

[72] Y. Lei, M. Rahim, Q. Ng, and T. Segura, "Hyaluronic acid and fibrin hydrogels with concentrated DNA/PEI polyplexes for local gene delivery," Journal of Controlled Release, vol. 153, no. 3, pp. 255-261, 2011.

[73] S. Siegman, N. F. Truong, and T. Segura, "Encapsulation of PEGylated low-molecular-weight PEI polyplexes in hyaluronic acid hydrogels reduces aggregation," Acta Biomaterialia, vol. 28, pp. 45-54, 2015.

[74] T. Tokatlian, C. Cam, S. N. Siegman, Y. Lei, and T. Segura, "Design and characterization of microporous hyaluronic acid hydrogels for in vitro gene transfer to mMSCs," Acta Biomaterialia, vol. 8, no. 11, pp. 3921-3931, 2012.

[75] S. Gojgini, T. Tokatlian, and T. Segura, "Utilizing cell-matrix interactions to modulate gene transfer to stem cells inside hyaluronic acid hydrogels," Molecular Pharmaceutics, vol. 8, no. 5, pp. 1582-1591, 2011.

[76] J. Bonadio, E. Smiley, P. Patil, and S. Goldstein, "Localized, direct plasmid gene delivery in vivo: prolonged therapy results in reproducible tissue regeneration," Nature Medicine, vol. 5, no. 7, pp. 753-759, 1999.

[77] M. Cucchiarini and H. Madry, "Gene therapy for cartilage defects," Journal of Gene Medicine, vol. 7, no. 12, pp. 1495-1509, 2005.

[78] H. Madry, P. Orth, and M. Cucchiarini, "Gene therapy for cartilage repair," Cartilage, vol. 2, no. 3, pp. 201-225, 2011.

[79] S. C. Ghivizzani, E. R. Lechman, R. Kang et al., "Direct adenovirus-mediated gene transfer of interleukin 1 and tumor necrosis factor $\alpha$ soluble receptors to rabbit knees with experimental arthritis has local and distal anti-arthritic effects," Proceedings of the National Academy of Sciences of the United States of America, vol. 95, no. 8, pp. 4613-4618, 1998.

[80] D. D. Frisbie, S. C. Ghivizzani, P. D. Robbins, C. H. Evans, and C. W. McIlwraith, "Treatment of experimental equine osteoarthritis by in vivo delivery of the equine interleukin-1 receptor antagonist gene," Gene Therapy, vol. 9, no. 1, pp. 12-20, 2002.

[81] K. Gelse, K. von der Mark, T. Aigner, J. Park, and H. Schneider, "Articular cartilage repair by gene therapy using growth factorproducing mesenchymal cells," Arthritis and Rheumatism, vol. 48, no. 2, pp. 430-441, 2003.

[82] T. Oligino, S. C. Ghivizzani, D. Wolfe et al., "Intra-articular delivery of a herpes simplex virus IL-1Ra gene vector reduces inflammation in a rabbit model of arthritis," Gene Therapy, vol. 6, no. 10, pp. 1713-1720, 1999.

[83] J. M. Mason, A. S. Breitbart, M. Barcia, D. Porti, R. G. Pergolizzi, and D. A. Grande, "Cartilage and bone regeneration using geneenhanced tissue engineering," Clinical Orthopaedics and Related Research, no. 379, pp. S171-S178, 2000.

[84] E. Gouze, R. Pawliuk, J.-N. Gouze et al., "Lentiviral-mediated gene delivery to synovium: potent intra-articular expression with amplification by inflammation," Molecular Therapy, vol. 7, no. 4, pp. 460-466, 2003.

[85] C. H. Evans, P. D. Robbins, S. C. Ghivizzani et al., "Clinical trial to assess the safety, feasibility, and efficacy of transferring a potentially anti-arthritic cytokine gene to human joints with rheumatoid arthritis," Human Gene Therapy, vol. 7, no. 10, pp. 1261-1280, 1996.

[86] M. Cucchiarini, H. Madry, C. Ma et al., "Improved tissue repair in articular cartilage defects in vivo by rAAV-mediated 
overexpression of human fibroblast growth factor 2," Molecular Therapy, vol. 12, no. 2, pp. 229-238, 2005.

[87] M. Cucchiarini, T. Thurn, A. Weimer, D. Kohn, E. F. Terwilliger, and H. Madry, "Restoration of the extracellular matrix in human osteoarthritic articular cartilage by overexpression of the transcription factor SOX9," Arthritis \& Rheumatism, vol. 56, no. 1, pp. 158-167, 2007.

[88] M. R. Pagnotto, Z. Wang, J. C. Karpie, M. Ferretti, X. Xiao, and C. R. Chu, "Adeno-associated viral gene transfer of transforming growth factor- $\beta 1$ to human mesenchymal stem cells improves cartilage repair," Gene Therapy, vol. 14, no. 10, pp. 804813, 2007.

[89] H. Madry, G. Kaul, D. Zurakowski, G. Vunjak-Novakovic, and M. Cucchiarini, "Cartilage constructs engineered from chondrocytes overexpressing IGF-I improve the repair of osteochondral defects in a rabbit model," European Cells and Materials, vol. 25, pp. 229-247, 2013.

[90] E. Lubberts, L. A. B. Joosten, M. Chabaud et al., "IL-4 gene therapy for collagen arthritis suppresses synovial IL-17 and osteoprotegerin ligand and prevents bone erosion," The Journal of Clinical Investigation, vol. 105, no. 12, pp. 1697-1710, 2000.

[91] J.-H. Jang, T. L. Houchin, and L. D. Shea, "Gene delivery from polymer scaffolds for tissue engineering," Expert Review of Medical Devices, vol. 1, no. 1, pp. 127-138, 2004.

[92] R. J. Levy, C. Song, S. Tallapragada et al., "Localized adenovirus gene delivery using antiviral IgG complexation," Gene Therapy, vol. 8, no. 9, pp. 659-667, 2001.

[93] R. M. Schek, S. J. Hollister, and P. H. Krebsbach, "Delivery and protection of adenoviruses using biocompatible hydrogels for localized gene therapy," Molecular Therapy, vol. 9, no. 1, pp. 130138, 2004.

[94] A. Breen, P. Strappe, A. Kumar, T. O’Brien, and A. Pandit, "Optimization of a fibrin scaffold for sustained release of an adenoviral gene vector," Journal of Biomedical Materials Research-Part A, vol. 78, no. 4, pp. 702-708, 2006.

[95] M. E. Kidd, S. Shin, and L. D. Shea, "Fibrin hydrogels for lentiviral gene delivery in vitro and in vivo," Journal of Controlled Release, vol. 157, no. 1, pp. 80-85, 2012.

[96] C. Alvarez-Lorenzo, A. Rey-Rico, A. Sosnik, P. Taboada, and A. Concheiro, "Poloxamine-based nanomaterials for drug delivery," Frontiers in Bioscience, vol. 2, no. 2, pp. 424-440, 2010.

[97] C. Alvarez-Lorenzo, A. Sosnik, and A. Concheiro, "PEO-PPO block copolymers for passive micellar targeting and overcoming multidrug resistance in cancer therapy," Current Drug Targets, vol. 12, no. 8, pp. 1112-1130, 2011. 


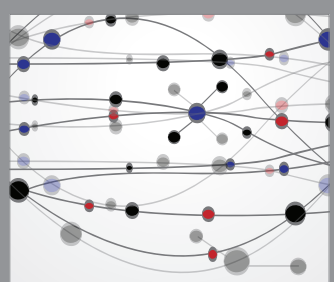

The Scientific World Journal
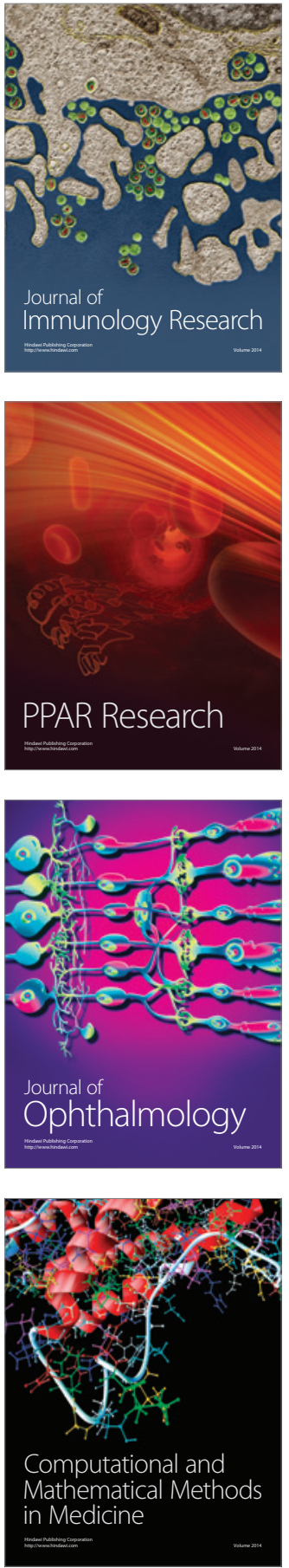

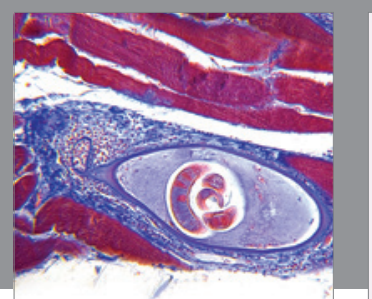

Gastroenterology Research and Practice

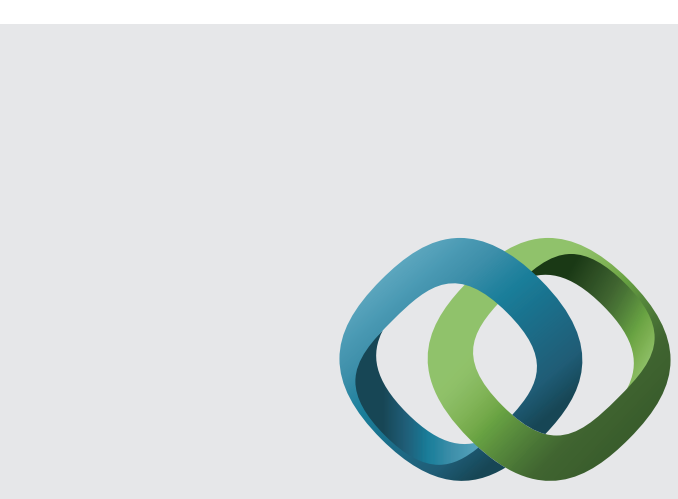

\section{Hindawi}

Submit your manuscripts at

http://www.hindawi.com
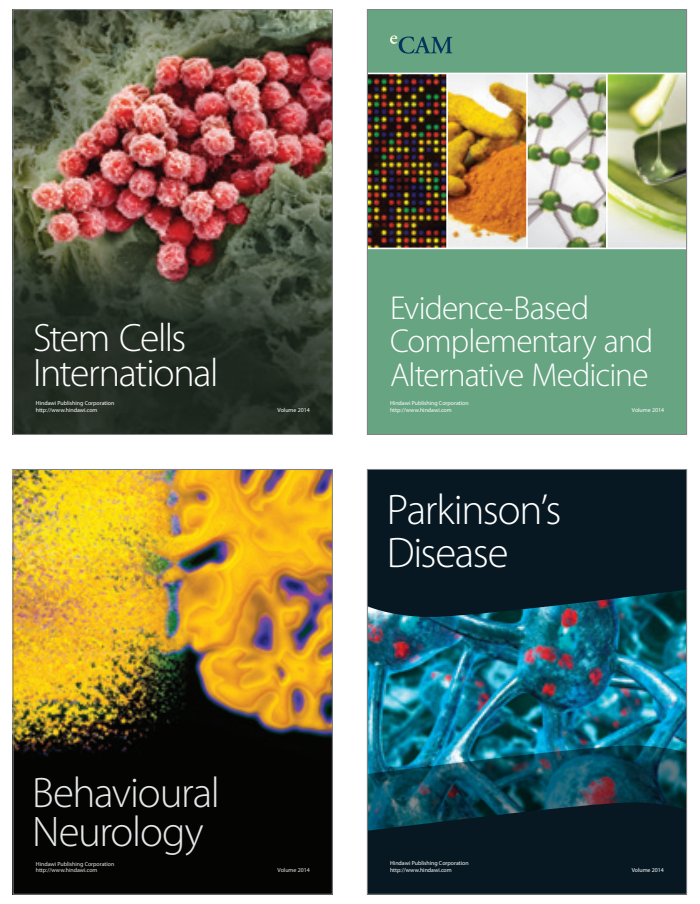
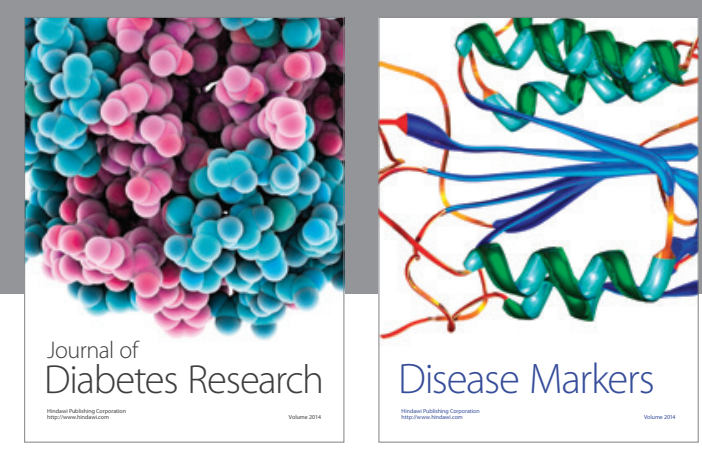

Disease Markers
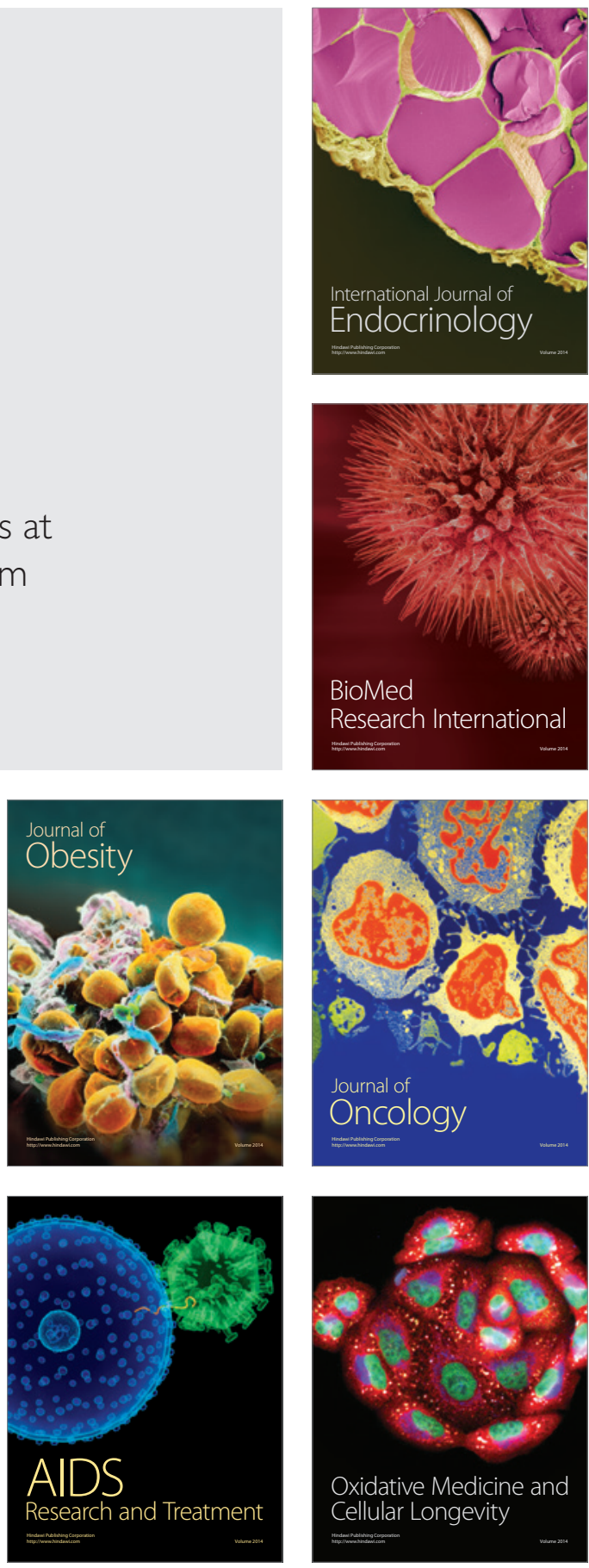